ThE ASTROPHYSICAL JoURNAL, 243:369-380, 1981 January 15

( 1981 . The American Astronomical Society. All rights reserved. Printed in U.S.A.

\title{
INFRARED AND OPTICAL OBSERVATIONS OF THE HYDROGEN LINES IN QUASARS
}

\author{
B. T. Soifer, G. Neugebauer, J. B. OKe, AND K. Matthews \\ Palomar Observatory, California Institute of Technology, Pasadena, California \\ Received 1980 May 19; accepted 1980 July 22
}

\begin{abstract}
Infrared and optical spectrophotometric observations of the hydrogen lines in a sample of 16 quasars are reported. Combining these observations with previously published observations of hydrogen lines in quasars brings to 12 the number of quasars for which the $\mathrm{P} \alpha / \mathrm{H} \alpha / \mathrm{H} \beta$ line ratios have been measured and brings to nine the number of quasars for which the $\mathrm{L} \alpha / \mathrm{H} \alpha$ line ratio has been measured.

The $\mathrm{P} \alpha / \mathrm{H} \alpha$ ratios in low-redshift quasars are distributed around the case $\mathrm{B}$ value with a tendency toward values lower than that predicted by case $\mathrm{B}$. The $\mathrm{H} \alpha / \mathrm{H} \beta$ values in these same quasars are generally greater than the case $B$ value. The trend in the $\mathrm{P} \alpha / \mathrm{H} \alpha / \mathrm{H} \beta$ ratios is for the $\mathrm{P} \alpha / \mathrm{H} \alpha$ ratio to decrease as $\mathrm{H} \alpha / \mathrm{H} \beta$ increases. The decrease of the $\mathrm{P} \alpha / \mathrm{H} \alpha$ ratio with increasing $\mathrm{H} \alpha / \mathrm{H} \beta$ ratio is the most significant correlation found from the present data that any valid model of the line-emitting regions must explain.

The low values of the $\mathrm{L} \alpha / \mathrm{H} \alpha$ compared to the case B value are confirmed for a large sample of high-redshift quasars. $\mathrm{L} \alpha$ is apparently destroyed rather than $\mathrm{H} \alpha$ being enhanced.

We conclude that reddening external to the emission-line regions cannot satisfactorily explain all the observed hydrogen line ratios. The current observations do not support the model of internal dust destroying resonantly scattered $\mathrm{L} \alpha$, but such a model cannot be ruled out on the basis of the present data. Models of the emission-line regions that apply radiative transfer and collisional excitation effects in the lines appear to be necessary to explain the current observations.
\end{abstract}

Subject headings: infrared: spectra - quasars

\section{INTRODUCTION}

The study of the emission-line spectra of quasars and active galactic nuclei has undergone a significant resurgence recently with the publication of quantitative optical, infrared, and ultraviolet spectroscopic observations. One of the major results of these observations, found to be true both in quasars and active galactic nuclei (e.g., Davidsen, Hartig, and Fastie 1977; Davidsen and Hartig 1978; Hyland, Becklin, and Neugebauer 1978) and anticipated by the composite quasar spectral analysis of Baldwin (1977a), has been the determination that the hydrogen line fluxes substantially disagree with the predictions of the early models of the quasar emission-line regions (Davidson 1972; Scargle, Caroff, and Tarter 1974).

Because of the fundamental problem posed by this discrepancy, a great deal of theoretical analysis has been performed to discover its solution. Thus far, two classes of physical processes have been suggested to account for the observed discrepancy. One involves the effects of radiative transfer and/or collisional excitation in the high-density regions where the $\mathrm{H} \mathrm{I}$ lines are formed (Krolik and McKee 1978; Zirin 1978; Kwan and Krolik 1979; Canfield and Puetter 1980a). The other involves reddening within or external to the lineemitting region (London 1979; Netzer and Davidson 1979; Shuder and MacAlpine 1979). An overview of theoretical treatments is provided in the review by Davidson and Netzer (1979).

In this paper we report combined visual and infrared observations of the hydrogen lines in a significant sample of low- and high-redshift quasars. The intent of this program has been to study the hydrogen line ratios in a sufficiently large sample of these objects to allow meaningful observational constraints to be placed on the models of the emission-line regions.

The hydrogen lines studied are $\mathrm{L} \alpha, \mathrm{H} \alpha, \mathrm{H} \beta$, and $\mathrm{P} \alpha$. Observationally, the study divides into two distinct programs. In low-redshift quasars, with redshifts $z$ in the range $0.07 \leq z \leq 0.30, \quad \operatorname{P} \alpha\left(\lambda_{\text {rest }}=1.8751 \mu \mathrm{m}\right)$ is accessible in the atmospheric window from $2.0 \mu \mathrm{m}$ to $2.45 \mu \mathrm{m}$, while $\mathrm{H} \alpha$ and $\mathrm{H} \beta$ can be observed in the visual. For quasars where $z>1.63, \mathrm{~L} \alpha$ enters the visual band. For $z$ in the range $1.63-1.74, \mathrm{H} \alpha$ falls in the 1.65 $\mu \mathrm{m}$ atmospheric window, while for $z$ between 2.05 and 2.7, $\mathrm{H} \alpha$ falls in the $2.2 \mu \mathrm{m}$ window. For $3.1 \leq z<3.9$, $\mathrm{H} \beta$ falls in the $2.2 \mu \mathrm{m}$ window, and the $\mathrm{L} \alpha / \mathrm{H} \beta$ ratio is directly observable. 
TABLE 1

NeW Infrared and Optical Observations of Quasars

\begin{tabular}{|c|c|c|c|c|}
\hline \multirow[b]{2}{*}{ ОвЈеСт } & \multirow{2}{*}{$\begin{array}{c}\text { REDSHFT } \\
(z)\end{array}$} & \multicolumn{2}{|c|}{ Date of ObSERVATION } & \multirow[b]{2}{*}{ Notes } \\
\hline & & Infrared & Visual & \\
\hline $0026+12(P G)$. & 0.147 & 1979 Oct & $1976 \mathrm{Dec}$ & $a, b$ \\
\hline $0101+13$ (PHL 957). & 2.69 & $1979 \mathrm{Jul}$ & 1970 Nov & b \\
\hline $0205+02$ (NAB) $\ldots \ldots \ldots \ldots$ & 0.155 & 1977 Oct & $1978 \mathrm{Feb}$ & b \\
\hline 0837-12 (PKS, 3C 206).... & 0.200 & $1980 \mathrm{Mar}$ & 1978 May & b \\
\hline $0906+48$ (PG) . . . . & 0.118 & 1977 Oct & $1978 \mathrm{Feb}$ & b \\
\hline $1012+25$ (Ton 490).... & 1.631 & 1979 Jan & 1979 Jun & \\
\hline $1211+14$ (PG) $\ldots \ldots$ & 0.085 & 1979 Jan & 1979 May & a \\
\hline $1307+08(\mathrm{PG}) \ldots$ & 0.160 & 1979 Jan & 1979 May & a \\
\hline $1346-03 \ldots \ldots$ & 2.344 & 1979 Jan & 1979 May & \\
\hline $1519+22(P G) \ldots$ & 0.140 & 1978 Jun & $1978 \mathrm{Jul}$ & \\
\hline $1612+25($ Ton 256$) \ldots \ldots \ldots$ & 0.131 & 1978 May & $1969 \mathrm{Jul}$ & b \\
\hline $1623+26$ (SW 77) $\ldots \ldots \ldots$ & 2.54 & $1978 \mathrm{Jul}$ & 1978 May & c \\
\hline $1721+34(4 C 34.47) \ldots \ldots$ & 0.205 & $1979 \mathrm{Jul}$ & 1979 Oct & \\
\hline $2126-15(\mathrm{PKS}) \ldots \ldots$ & 3.27 & $1978 \mathrm{Jul}$ & $1978 \mathrm{Jul}$ & \\
\hline $2141+17(\mathrm{PKS}$, OX 169) $\ldots$ & 0.213 & 1978 Jun & $1978 \mathrm{Jul}$ & \\
\hline $2201+31(4 C 31.63)$ & 0.297 & $1978 \mathrm{Jul}$ & 1976 Dec & b \\
\hline
\end{tabular}

a Infrared observations made at $2.5 \mathrm{~m}$ Hooker Telescope at Mount Wilson.

bVisual observations previously published in Neugebauer et al. 1979.

${ }^{\mathrm{c}}$ From Sramek and Weedman 1978.

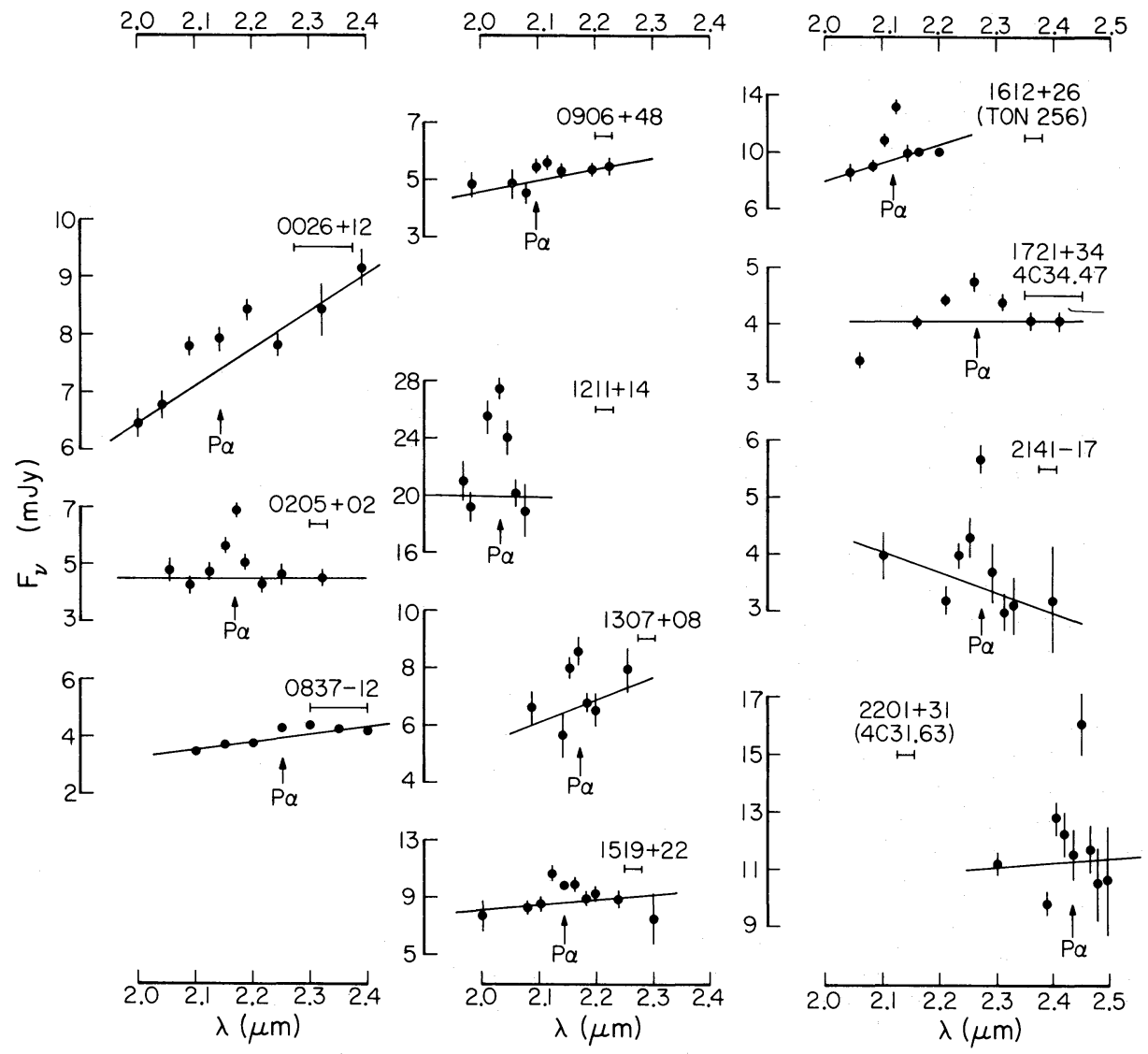

Fig. 1. - The near-infrared spectra of low-redshift quasars in the vicinity of the Paschen $\alpha$ line. The associated flux density scale, in units of $\mathrm{mJy}$, is at the left of each spectrum. The instrumental full width at half-maximum is indicated by the horizontal bar below the object name. In each case the expected wavelength of Pa, from the optically determined redshifts, is noted in the spectrum. The straight line in each spectrum is the continuum used for that object to calculate the line flux in Pa. 


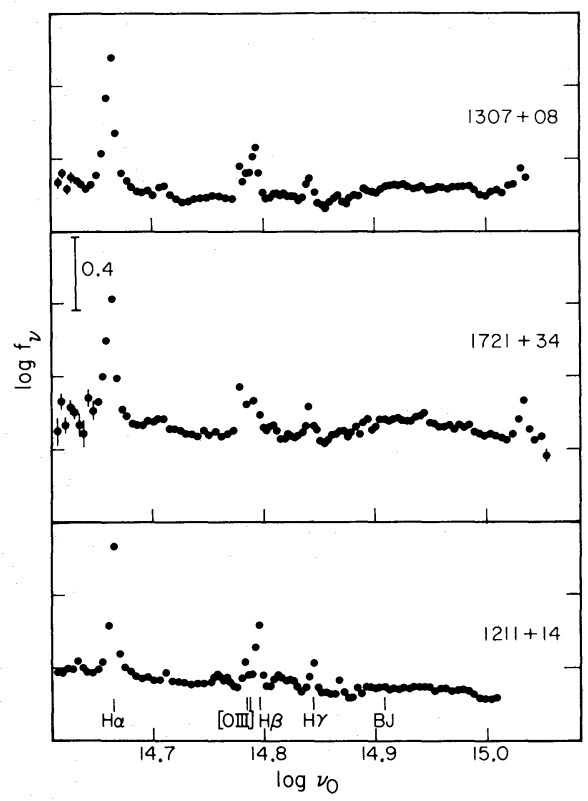

Fig. 2.-Optical spectrophotometric observations of three low-redshift quasars that illustrate the range of Balmer decrements observed in the low-redshift quasars. The data are plotted in the rest frame of the quasars. The wavelength of the hydrogen lines $\mathrm{H} \alpha, \mathrm{H} \beta$, and $\mathrm{H} \gamma$, as well as the Balmer continuum limit and [O III] $\lambda \lambda 4959,5007$, are marked in the figure. The quasar $1307+08$ illustrates a large decrement $(\mathrm{H} \alpha / \mathrm{H} \beta=5.5), 1721+34$ a moderate decrement $(\mathrm{H} \alpha / \mathrm{H} \beta=4.0$ before correction for galactic reddening), and $1211+14$ a small decrement $(\mathrm{H} \alpha / \mathrm{H} \beta=3.4)$.

\section{OBSERVATIONS}

The observations reported here consist of both visual and infrared spectrophotometry of quasars selected on the basis of being bright and having redshifts in the appropriate range. All the visual observations were obtained with the multichannel spectrometer on the 5.1 $\mathrm{m}$ Hale telescope. Some of these observations have been previously reported by Neugebauer et al. (1979) and details of the observations are given therein.

The infrared observations were obtained with two cryogenically cooled circular variable infrared filterwheel spectrometers on the $5.1 \mathrm{~m}$ Hale telescope on Palomar Mountain and, in the case of several of the brightest objects, on the $2.5 \mathrm{~m}$ Hooker telescope on Mount Wilson. The resolution available for the infrared observations was either $\Delta \lambda / \lambda \approx 0.013$ or $\Delta \lambda / \lambda \approx 0.05$, corresponding to $3900 \mathrm{~km} \mathrm{~s}^{-1}$ or $15,000 \mathrm{~km} \mathrm{~s}^{-1}$, respectively. All the P $\alpha$ observations except those of PG $0026+129,0837-12,(3 \mathrm{C} 206)$, and 1720+34 (4C 34.47) were made with the higher-resolution instrument, while all the observations of $\mathrm{H} \alpha$ in the high-redshift quasars were made with the lower-resolution instrument. Although hydrogen lines in quasars are generally broader than $5000 \mathrm{~km} \mathrm{~s}^{-1}$ (see, e.g., Baldwin 1975), no attempt has been made to obtain line widths from the present infrared observations.

\section{RESULTS-LINE FLUXES}

A compilation of the visual and infrared observations is given in Table 1 . For the most part, the separation in time between the visual and infrared observations of any object was shorter than a few months, but in some cases the time interval was longer, and in two notable cases, $1612+26$ (Ton 256) and $0101+13$ (PHL 957), the visual and infrared observations were separated by almost 10 years. While there is some worry about comparing such widely separated observations, there is little or no indication that these quasars have varied significantly over this interval. In the case of $0101+13$ there is evidence against variability, since both the equivalent widths of $\mathrm{L} \alpha$ and $\mathrm{C} \mathrm{IV}$ and the continuum levels agree, to within $20 \%$, with the observations five years later reported by Baldwin $(1977 b)$. For $1612+26$ the continuum flux at $5500 \AA$

TABLE 2

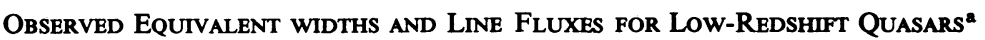

\begin{tabular}{|c|c|c|c|c|c|c|c|c|c|c|c|}
\hline \multirow[b]{2}{*}{ ОвЈеСт } & \multicolumn{3}{|c|}{$\mathbf{H} \alpha$} & \multicolumn{3}{|c|}{$\mathbf{H} \beta$} & \multicolumn{3}{|c|}{ [O II] } & \multicolumn{2}{|c|}{$\mathbf{P} \alpha$} \\
\hline & $\mathrm{EW}$ & $j$ & Unc. & EW & $j$ & Unc. & EW & $j$ & Unc. & EW & $j$ \\
\hline $0026+12$ & 430 & 81 & b & 77 & 20 & c & 36 & 9 & e & 140 & $7 \pm 2$ \\
\hline $0205+02$ & 470 & 53 & b & 87 & 16 & c & 48 & 8 & d & 190 & $5.5 \pm 0.5$ \\
\hline $0837-12$ & 650 & 30 & c & 130 & 6.8 & c & 110 & 6 & $d$ & 208 & $4.9 \pm 1.0$ \\
\hline $0906+48$ & 430 & 24 & b & 62 & 5 & c & 36 & 2.9 & c & 30 & $1.0 \pm 0.6$ \\
\hline $1211+14$ & 450 & 220 & b & 83 & 66 & b & 31 & 23 & c & 150 & $22 \pm 2$ \\
\hline $1307+08$ & 700 & 90 & c & 77 & 16 & c & 71 & 14 & c & 80 & $3.4 \pm 0.7$ \\
\hline $1519+22$ & 410 & 34 & b & 110 & 12 & c & 32 & 3.3 & c & 100 & $5.5 \pm 1.5$ \\
\hline $1612+26$ & 825 & 103 & b & 170 & 30 & c & 176 & 29 & c & 110 & $7 \pm 1$ \\
\hline $1721+34$ & 710 & 65 & b & 110 & 16 & c & 94 & 13 & c & 170 & $4.1 \pm 0.6$ \\
\hline $2141+17$ & 365 & 32 & c & 37 & 5 & c & 24 & 3.1 & c & 170 & $3.5 \pm 0.5$ \\
\hline $2201+31$ & 640 & 71 & b & 140 & 24 & c & 24 & 4 & d & $<62$ & $<3.5$ \\
\hline
\end{tabular}

a Equivalent widths (EW) in $\AA$ and line fluxes $(j)$ in $10^{-17} \mathrm{Wm}^{-2}$ reported in observers frame.

bUncertainty $<10 \%$.

${ }^{c}$ Uncertainty $10-25 \%$.

dUncertainty $25-50 \%$.

'Uncertainty $>50$. 
measured in 1979 July agrees with that measured in 1969 July to within 5\%, again providing evidence against variability in this quasar. It is also the case that even in quasars where the continuum varies, there is evidence that the line strengths do not vary (see, e.g., Netzer et al. 1979; Davidson and Netzer 1979, and references therein). It is therefore reasonable to compare the line fluxes measured at such a large separation in time.

The spectra of the low-redshift quasars in the region of $\mathrm{P} \alpha$ are shown in Figure 1, while a representative selection of visual spectra of the low-redshift quasars is shown in Figure 2. The expected wavelength of $P \alpha$ is shown for all the quasars in Figure 1 as well as a linear fit to the continuum for each quasar; the latter was derived using the data points well away from the $\mathrm{P} \alpha$ line. The equivalent widths of the $\mathrm{P} \alpha$ line are given in Table 2, as are the observed fluxes in the line. Both the equivalent widths and line fluxes are as observed.

In the case of the visual lines, the equivalent width of each line was measured with respect to a continuum established from the observed flux minima in the vicinity of that line. The observed fluxes in the $\mathrm{H} \alpha, \mathrm{H} \beta$, and [O III] lines, derived from the equivalent widths, are included in Table 2. It has been assumed, based on the work of Baldwin (1975), that there is negligible contribution of [N II] $\lambda \lambda 6548,6584$ to the flux from $\mathrm{H} \alpha$. In the cases where the redshifts were sufficient to cause the atmospheric $A$ band to significantly affect the $\mathrm{H} \alpha$ flux, i.e., for $0837-12$ and $1307+08$, the uncertainty in the $\mathrm{H} \alpha$ line flux was increased, even though a first-order correction for this effect was made in the data reduction. A symmetric $\mathrm{H} \beta$ line profile was assumed in separating $\mathrm{H} \beta$ from [O III] $\lambda \lambda 4959,5007$ line fluxes. For each measurement, the uncertainty in the line flux and equivalent width listed in Table 2 is a combination of the statistical uncertainty in the measurement, the uncertainty in establishing the continuum, and, in the case of $\mathbf{H} \beta$ and [O $\mathrm{III}]$, the estimated uncertainty associated with this separation.

The infrared spectrophotometry of the high-redshift quasars is shown in Figure 3, with the redshifted Balmer lines indicated. Visual spectrophotometry of most of these quasars has been previously published (see, e.g., Osmer and Smith 1977; Baldwin 1975; Neugebauer et al. 1979). The hydrogen line strengths in these quasars, both equivalent widths and emission line fluxes, were derived in the same fashion as described previously, and are given in Table 3. In the case of $\mathrm{L} \alpha$, the equivalent widths and line fluxes derived from the multichannel spectrophotometer data are given without attempting to correct for $\mathrm{L} \alpha$ absorption. Since the lines are so broad, the correction for $N$ v $\lambda 1240$ blending with $\mathrm{L} \alpha$ becomes somewhat uncertain. $\mathrm{N} v$ is commonly assumed to be one-fourth of $\mathrm{L} \alpha$ (see, e.g., Baldwin 1977b), and we have used this assumed ratio throughout in deriving $\mathrm{L} \alpha$ line fluxes.

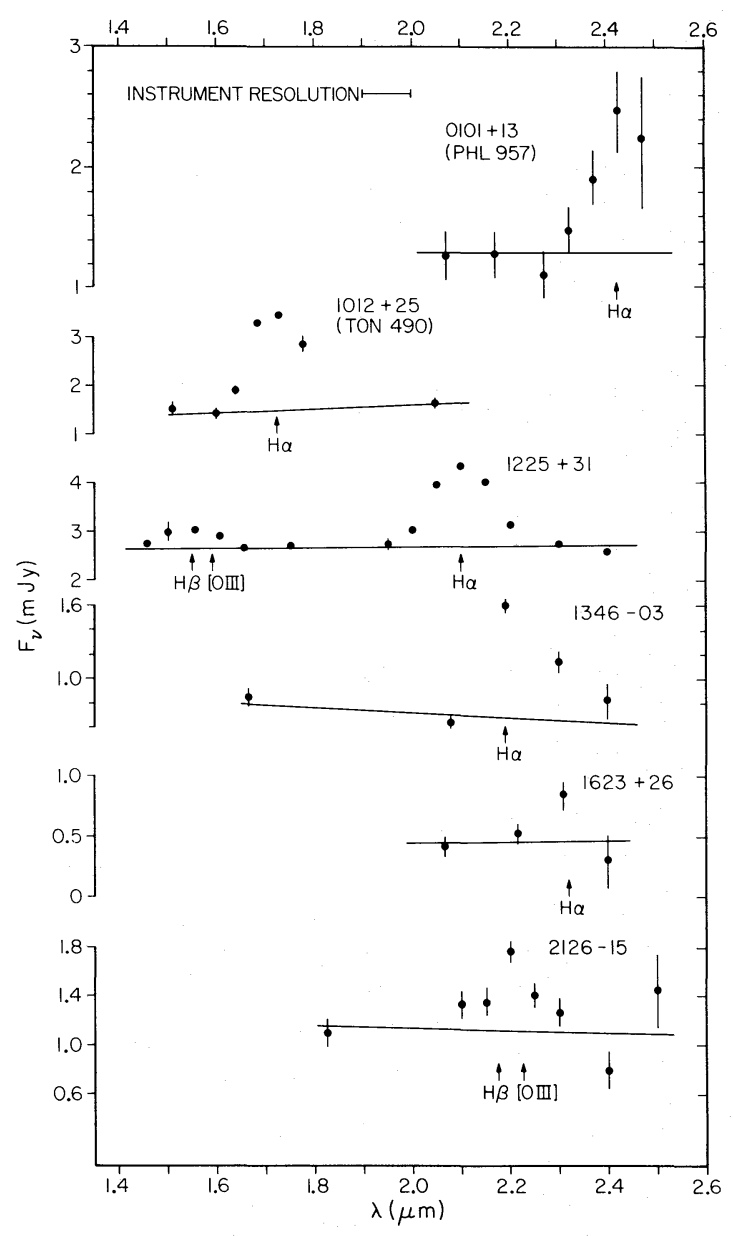

FIG. 3.-The near-infrared spectra of the high-redshift quasars in the vicinity of the $\mathrm{H} \alpha$, and in two cases, $\mathrm{H} \beta+[\mathrm{O} \mathrm{mI}]$ lines. The instrumental full width at half-maximum is illustrated by the horizontal bar at the top of the plot. The expected wavelength of the appropriate line, derived from the optically determined redshift, is given in each spectrum. The data for $1225+31$ are taken from Soifer et al. (1979).

In the case of two of the high-redshift quasars, special procedures were required to derive the line fluxes shown in Table 3. In 1012+25 (Ton 490), the redshift is sufficient to make only half of the $\mathrm{L} \alpha$ line accessible in the visual window. For this reason, the uncertainty in the $\mathrm{L} \alpha+\mathrm{N} v$ line flux was increased to $25 \%$. In PKS 2126-15, the redshift was such that $\mathrm{H} \beta$ and [O III] were shifted into the $2.2 \mu \mathrm{m}$ atmospheric window. The $\mathrm{H} \alpha$ flux was estimated by assuming that the $\mathrm{H} \alpha / \mathrm{H} \beta /[\mathrm{O} \text { III }]^{1}$ flux ratios in PKS 2126-15 were the same as the average values determined from the low-redshift quasars; $\mathrm{H} \alpha / \mathrm{H} \beta /\left[\begin{array}{ll}\mathrm{O} & \mathrm{III}\end{array}\right]=3.8 / 1.0 / 0.5$. Since the composite spectrum approach has proved so successful in establishing the $\mathrm{L} \alpha / \mathrm{H} \alpha$ ratio, it was felt

\footnotetext{
${ }^{1}$ Throughout this paper we will use notation such as, e.g., $\mathrm{H} \alpha / \mathrm{H} \beta$, to indicate the ratio of the line fluxes.
} 
TABLE 3

ObSeRved Equivalent Widths and Line FluXes in High-Redshift Quasars ${ }^{a}$

\begin{tabular}{lccccccc}
\hline \hline & \multicolumn{3}{c}{$\mathrm{H} \alpha$} & \multicolumn{2}{c}{$\mathrm{L} \alpha+\mathrm{N} \mathrm{v}$} & \multicolumn{2}{c}{ C IV } \\
\cline { 2 - 8 } OвJест & EW & $j$ & EW & $j$ & EW & $j$ & NotES \\
\hline $0101+13 \ldots$ & 1030 & $7 \pm 2.5$ & 130 & $15 \pm 3$ & 56 & $4.5 \pm 1$ & $\mathrm{~b}$ \\
$1012+25 \ldots$ & 1660 & $25 \pm 5$ & 360 & $26 \pm 6$ & 140 & $13 \pm 2$ & \\
$1346-03 \ldots$ & 2100 & $9 \pm 1$ & 300 & $16 \pm 2$ & 120 & $5 \pm 1$ & \\
$1623+26 \ldots$ & 810 & $2.1 \pm 0.8$ & 130 & $10 \pm 2.5$ & 39 & $1.9 \pm 0.2$ & \\
$2126-15 \ldots$ & $\ldots$ & 12.5 & 190 & $11 \pm 3$ & 26 & $1.2 \pm 0.4$ & $\mathrm{c}$ \\
\hline
\end{tabular}

a Equivalent widths (EW) in $\AA$ and line fluxes $(j)$ in $10^{-17} \mathrm{Wm}^{-2}$ reported in observers' frame. ${ }^{b} \mathrm{~L} \alpha$ and $\mathrm{C}$ iv from Neugebauer et al. 1979.

${ }^{c} \mathrm{H} \alpha$ derived as described in text from $j(\mathrm{H} \beta+[\mathrm{O} \mathrm{III}])=5 \pm 2 \times 10^{-17} \mathrm{Wm}^{-2}$. Uncertainty is at least a factor of 2 .

that it is a reasonable approach to estimating the $\mathrm{H} \alpha$ flux. Clearly the derived value has a large uncertainty, no less than a factor of 2 .

\section{RESULTS}

The observational results in the form of various line ratios are given in Tables 4 and 5 . All the line ratios have been corrected for the effects of interstellar extinction in the Galaxy following the precepts of Sandage (1972). In this model, the extinction for galactic latitudes $|b|$ greater than $50^{\circ}$ is negligible. The tables also include all the available published values of the line ratios under discussion for quasars.

The new line ratios reported in Tables 4 and 5 can be compared, in several instances, with previously published results. The $\mathrm{P} \alpha$ line flux reported here for $0026+$ 129 is $50 \%$ lower than the value of Puetter et al. (1978) as revised by Puetter (private communication). In addition, Puetter et al. (1981) have measured infrared line fluxes of seven of the quasars in Table 1; the agreement with the results in Tables 2 and 3 is good.

Visual observations of several of the quasars have been previously reported. As mentioned above, the agreement of the present $\mathrm{L} \alpha$ and $\mathrm{C}$ IV line fluxes in $0101+13$ (PHL 957) with the data of Baldwin (1977b) is excellent. The present observations of $1012+25$ (Ton 490) agree with those of Baldwin (1977b) and Baldwin and Netzer (1978) in the equivalent widths of $L \alpha$ and C IV, and in the continuum flux to within $20 \%$. In the case of 1346-03, the equivalent width of $\mathrm{L} \alpha$ agrees with that reported by Osmer and Smith (1977) to within $20 \%$, while the C IV equivalent widths differ by $40 \%$. Observations of PKS 0837-12 reported in Baldwin (1975) give a ratio [O $\mathrm{III}] / \mathrm{H} \beta \sim 1.3$, compared with the present results of $0.8 \pm 0.4$. Grandi and Phillips (1979) report $\sim 3.8$ for the total $\mathrm{H} \alpha+\mathrm{N}$ II $/ \mathrm{H} \beta$ (broad + narrow) in good agreement with the value reported here of $3.7 \pm 0.4$. They report $\mathrm{O}$ III $/ \mathrm{H} \beta \sim 0.74$ compared to $0.8 \pm 0.2$ reported here. It is interesting to note

TABLE 4

LINE RATIOS IN LOW-z QUASARs ${ }^{a}$

\begin{tabular}{lcccccr}
\hline \hline Object & $\begin{array}{c}|b| \\
(\mathrm{deg})\end{array}$ & $\begin{array}{c}(B-V) \\
(\mathrm{mag})\end{array}$ & $\mathrm{H} \alpha / \mathrm{H} \beta$ & $\mathrm{P} \alpha / \mathrm{H} \alpha$ & {$[\mathrm{O} \mathrm{III]}] / \mathrm{H} \beta$} & $\alpha(\mathrm{P} \alpha, \mathrm{H} \beta)$ \\
\hline $0026+12 \ldots$ & 49 & 0.003 & $4.0^{\mathrm{c}}$ & $0.08 \pm 0.02$ & $0.4^{\mathrm{e}}$ & -0.73 \\
$0205+02 \ldots$ & 55 & $\ldots$ & $3.3^{\mathrm{c}}$ & $0.10 \pm 0.02$ & $0.5^{\mathrm{d}}$ & -0.61 \\
$0837-12 \ldots$ & 17 & 0.162 & $3.6^{\mathrm{c}}$ & $0.12 \pm 0.02$ & $0.8^{\mathrm{d}}$ & -0.91 \\
$0906+48 \ldots$ & 43 & 0.022 & $4^{\mathrm{c}}$ & $0.04 \pm 0.02$ & $0.58^{\mathrm{c}}$ & -1.25 \\
$1211+14 \ldots$ & 74 & $\ldots$ & $3.4^{\mathrm{c}}$ & $0.10 \pm 0.02$ & $0.35^{\mathrm{c}}$ & -0.75 \\
$1226+02^{\mathrm{b}} \ldots$ & 64 & $\ldots$ & $3.1^{\mathrm{c}}$ & $0.095 \pm 0.01$ & $<0.1$ & -0.88 \\
$1307+08 \ldots$ & 70 & $\ldots$ & $5.5^{\mathrm{c}}$ & $0.04 \pm 0.01$ & $0.9^{\mathrm{d}}$ & -0.78 \\
$1519+22 \ldots$ & 56 & $\ldots$ & $2.9^{\mathrm{c}}$ & $0.16 \pm 0.04$ & $0.3^{\mathrm{d}}$ & -1.27 \\
$1612+26 \ldots$ & 45 & 0.015 & $3.4^{\mathrm{c}}$ & $0.07 \pm 0.01$ & $1.0^{\mathrm{d}}$ & -1.18 \\
$1721+34 \ldots$ & 32 & 0.058 & $3.7^{\mathrm{c}}$ & $0.06 \pm 0.01$ & $0.8^{\mathrm{d}}$ & -0.53 \\
$2141+17 \ldots$ & 26 & 0.088 & $5.6^{\mathrm{c}}$ & $0.09 \pm 0.02$ & $0.6^{\mathrm{d}}$ & -0.38 \\
$2201+31 \ldots$ & 19 & 0.143 & $2.5^{\mathrm{c}}$ & $<0.04$ & 0.2 & -0.81 \\
\hline
\end{tabular}

${ }^{a}$ Line intensities corrected for galactic reddening using precepts of Sandage 1972.

${ }^{b} \mathrm{P} \alpha$ data from Grasdalen 1976, Puetter et al. 1978; $\mathrm{H} \alpha, \mathrm{H} \beta$ data from Neugebauer $e$ al. 1979.

'Uncertainty $10-25 \%$.

dUncertainty $25-50 \%$.

'Uncertainty $>50 \%$. 
TABLE 5

L $\alpha /$ H $\alpha$ Line RATIOS In QuAsars ${ }^{a}$

\begin{tabular}{cccccc}
\hline \hline Object & $\begin{array}{c}|b| \\
(\mathrm{deg})\end{array}$ & $\begin{array}{c}E(B-V) \\
(\mathrm{mag})\end{array}$ & $\mathrm{L} \alpha / \mathrm{H} \alpha$ & $\alpha(\mathrm{H} \alpha, \mathrm{L} \alpha)$ & Note \\
\hline $0026+12 \ldots$ & 49 & 0.003 & $1.2 \pm 0.4$ & -0.34 & $\mathrm{~b}$ \\
$0101+13 \ldots$ & 50 & 0.002 & $1.8(+1.0,-0.5)$ & -0.32 & \\
$0237-23 \ldots$ & 65 & $\ldots$ & $1.1 \pm 0.3$ & -0.68 & $\mathrm{c}$ \\
$1012+25 \ldots$ & 55 & $\ldots$ & $0.8 \pm 0.3$ & -0.95 & \\
$1225+31 \ldots$ & 84 & $\ldots$ & $0.8 \pm 0.2$ & -0.26 & $\mathrm{~d}$ \\
$1226+02 \ldots$ & 64 & $\ldots$ & $1.8 \pm 0.2$ & -0.54 & $\mathrm{e}$ \\
$1346-03 \ldots$ & 57 & $\ldots$ & $1.4 \pm 0.3$ & -0.48 & \\
$1623+26 \ldots$ & 43 & 0.022 & $4(+3,-2)$ & +0.07 & \\
$2126-15 \ldots$ & 42 & 0.025 & $0.7 \pm 0.6$ & -0.34 & \\
\hline
\end{tabular}

${ }^{a}$ Line intensities corrected for galactic reddening using precepts of Sandage 1972.

${ }^{b}$ Data from Baldwin et al. 1978.

'Data from Hyland et al. 1978.

¿Data from Soifer et al. 1979.

'Data from Boggess et al. 1979.

that the relation between continuum luminosity and C IV equivalent width found by Baldwin (1977b) holds reasonably well for the quasars $1346-03,1623+26$, and 2126-15.

\section{DISCUSSION}

The present observations bring to 12 the number of low-redshift quasars for which the $\mathrm{H} \alpha, \mathrm{H} \beta$, and $\mathrm{P} \alpha$ lines have been observed, and bring to nine the number of quasars where both $\mathrm{L} \alpha$ and $\mathrm{H} \alpha$ or $\mathrm{H} \beta$ have been observed. These numbers are sufficient to allow trends and correlations in the data to be sought in order to constrain the range of viable models of the quasar emission-line regions.

\section{a) Line Ratios}

The line ratios of Tables 4 and 5 are displayed in various ways in Figures 4 and 5. In these figures, and in the discussion that follows, we make the implicit assumption that the high- and low-redshift quasars share common properties, and that conclusions from one set of quasars can be applied directly to the other. At least in the case of the $\mathrm{L} \alpha$ and $\mathrm{H} \alpha$ lines this seems to be true as both high- and low-redshift quasars show the same ratios. This has not been shown to be the case for the $\mathrm{P} \alpha / \mathrm{H} \alpha / \mathrm{H} \beta$ ratios, since $\mathrm{P} \alpha$ has not been observed in any quasars with redshift greater than 0.3 .

Figure 4 shows the distribution of the line ratios in histogram form relative to the value the ratio would assume if pure radiative recombination theory obtained plus the assumption that the Lyman lines were all optically thick, i.e., case B. In the remainder of this paper the "case B" values will be taken as $\mathrm{L} \alpha / \mathrm{H} \alpha=12$, $\mathrm{H} \alpha / \mathrm{H} \beta=2.9$, and $\mathrm{P} \alpha / \mathrm{H} \beta=0.3$ (Brocklehurst 1972; Pengelly and Seaton 1964). Perhaps the most surprising property of these histograms is how uniform the distribution of ratios is over the observed ranges. The $\mathrm{H} \alpha / \mathrm{H} \beta$ ratio clearly deviates from the case $\mathrm{B}$ value. This deviation is well known and has been studied for large numbers of quasars by Baldwin (1975) and Neugebauer et al. (1979).

In Figure 4, the $\mathrm{P} \alpha / \mathrm{H} \alpha$ ratio also shows some substantial departures from the case B model, with a general trend to lower values than that predicted from case $B$. On the other hand, the $\mathrm{P} \alpha / \mathrm{H} \beta$ ratio seems to be nearly case B, with a large scatter. In Figure 5, it is seen that although many of the low-redshift quasars,

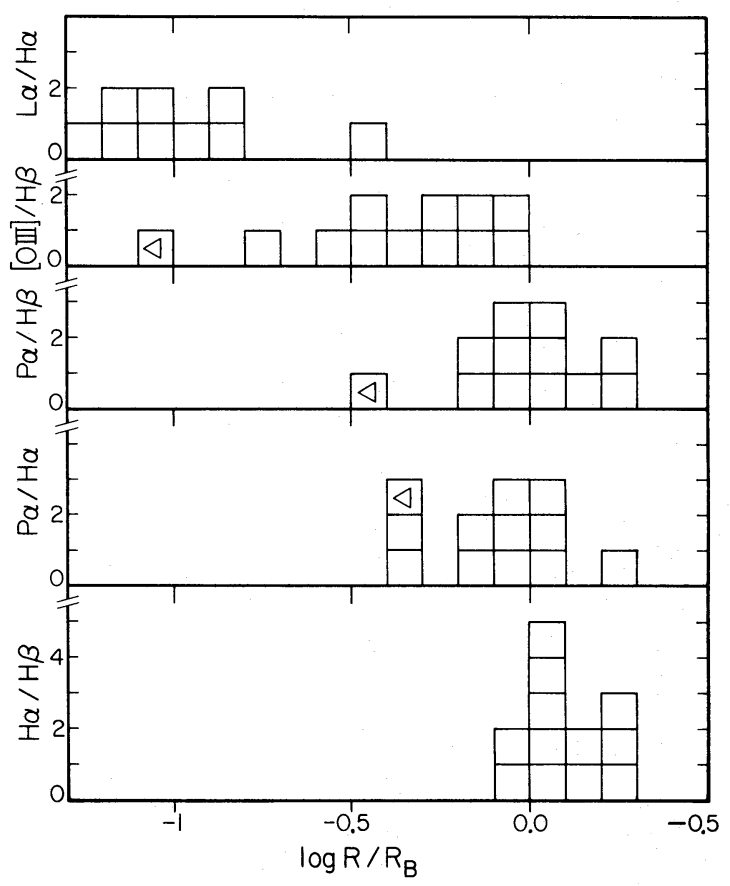

Fig. 4.-Histograms of numbers of occurrences of the observed line ratios normalized to the appropriate case $B$ value. The case $B$ ratios were taken to be $\mathrm{L} \alpha / \mathrm{H} \alpha=12, \mathrm{H} \alpha / \mathrm{H} \beta=2.9$, $\mathrm{P} \alpha / \mathrm{H} \alpha=0.1$, and $\mathrm{P} \alpha / \mathrm{H} \beta=0.3$. For [O $\mathrm{m}] / \mathrm{H} \beta$, the "case B" was taken to be 1 . The triangles represent observed values that are upper limits. 


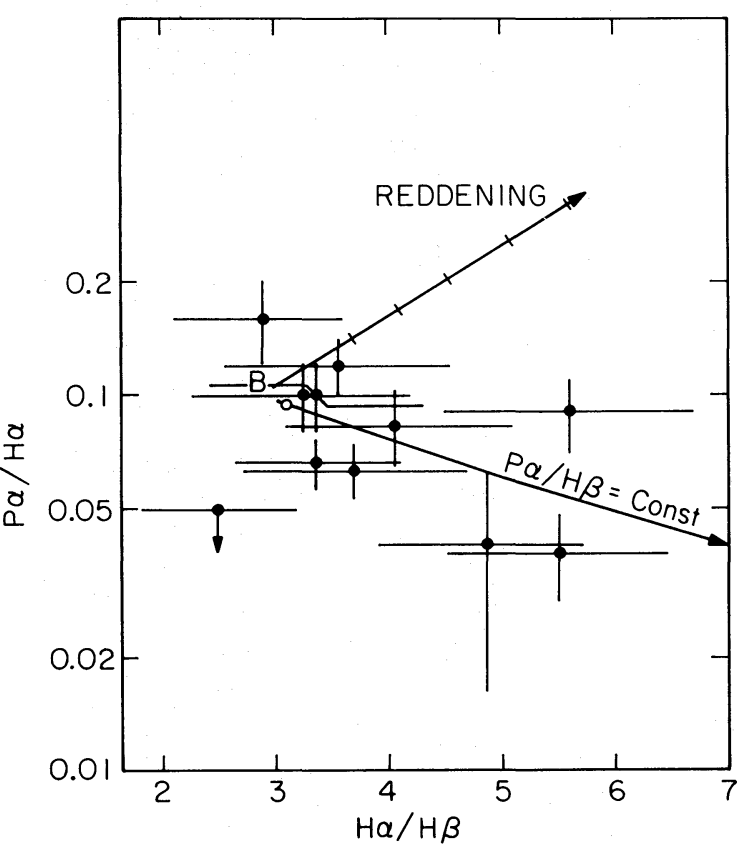

Fig. 5.-The observed $\mathrm{P} \alpha / \mathrm{H} \alpha$ line ratio plotted vs. the observed $\mathrm{H} \alpha / \mathrm{H} \beta$ line ratio for the quasars reported in Table 4 . The open circle is the observed position of $3 C 273$. The $B$ indicates the position of the case B result in this plot. The heavy arrow shows the effect of normal interstellar reddening on the case $B$ results. The successive tic marks indicate changes in $E(B-V)$ of 0.1 mag. The thin arrow indicates the deviations expected from the case B result due to collisional enhancement of $\mathrm{H} \alpha$ in the case of optically thin Balmer lines. In this case the $\mathrm{P} \alpha / \mathrm{H} \beta$ ratio remains constant. including 3C 273, group around the case B point in the $\mathrm{P} \alpha / \mathrm{H} \alpha$ versus $\mathrm{H} \alpha / \mathrm{H} \beta$ plane, there is a marked tendency for quasars with high $\mathrm{H} \alpha / \mathrm{H} \beta$ to have low $\mathrm{P} \alpha / \mathrm{H} \alpha$ ratios; the quasar $2141+17$ is a significant exception.

Figure 4 also shows that the newly observed highredshift quasars have qualitatively the same discrepancy between the observed value of $\mathrm{L} \alpha / \mathrm{H} \alpha$ and the ratio predicted by the simple case $B$ recombination theory, as that previously found in both high- and low-redshift quasars (Baldwin 1977a; Davidsen, Hartig, and Fastie 1977; Hyland, Becklin, and Neugebauer 1978; Soifer et al. 1979; Puetter, Smith, and Willner 1979). If the most uncertain values, those for 2126-15 and $1623+26$, are disregarded, the average value of $\mathrm{L} \alpha / \mathrm{H} \alpha$ for the high-redshift quasars is 1.2 , nearly the same as that for the two low-redshift quasars previously observed.

\section{b) $\mathrm{L} \alpha, \mathrm{H} \alpha$ Equivalent Widths}

Before the observed line ratios are compared with the various models put forth to explain them, it is useful to infer whether $\mathrm{L} \alpha$ is destroyed or $\mathrm{H} \alpha$ is enhanced in the sample of quasars where both lines are observed (Table 5), since this can give an insight into the physical processes at work in producing the observed ratios. This is done by comparing the observed equivalent widths of $\mathrm{L} \alpha$ and $\mathrm{H} \alpha$ with those estimated for each quasar based on the observed continuum slope. The quasars are assumed to consist of a central continuum source which ionizes the surrounding lineemitting clouds.

In Table 6 the relevant data are compiled. The observed equivalent widths of $\mathrm{L} \alpha$ are taken from Table

TABLE 6

Rest-Frame equivalent Widths of L $\alpha$ and $H \alpha$ In Quasars

\begin{tabular}{|c|c|c|c|c|c|c|}
\hline \multirow[b]{2}{*}{ ОвЈеСт } & \multicolumn{2}{|c|}{ EW of $\mathbf{L} \alpha$} & \multirow[b]{2}{*}{$\frac{\text { OBSERVED }}{\text { PrEDICTED }}$} & \multicolumn{2}{|c|}{ EW of $\mathrm{H} \alpha$} & \multirow[b]{2}{*}{$\frac{\text { OBSERVED }}{\text { PREDICTED }}$} \\
\hline & $\begin{array}{c}\text { Observed }^{\mathrm{a}} \\
\text { (๕) }\end{array}$ & $\begin{array}{l}\text { Predicted }^{\mathbf{b}} \\
\text { (๕) }\end{array}$ & & $\begin{array}{l}\text { Observed }^{\mathrm{a}} \\
\text { (A) }\end{array}$ & $\begin{array}{c}\text { Predicted }^{\mathrm{b}} \\
\text { (A) }\end{array}$ & \\
\hline $0026+12$. & 52 & 244 & 0.21 & 373 & 334 & 1.12 \\
\hline $0101+13 \ldots$ & 28 & 248 & 0.11 & 279 & 351 & 0.79 \\
\hline $0237-23 \ldots \ldots$ & 39 & 180 & 0.22 & 500 & 139 & 3.6 \\
\hline $1012+25 \ldots \ldots$ & 110 & 143 & 0.77 & 630 & 70 & 9.00 \\
\hline $1225+31 \ldots \ldots$ & 13 & 263 & 0.05 & 285 & 412 & 0.69 \\
\hline $1226+02 \ldots \ldots$ & 51 & 203 & 0.25 & 406 & 198 & 2.05 \\
\hline $1346-03 \ldots \ldots$ & 71 & 214 & 0.33 & 628 & 231 & 2.72 \\
\hline $1623+26 \ldots \ldots$ & 29 & 361 & 0.08 & 229 & 987 & 0.23 \\
\hline $2126-15 \ldots \ldots$ & 36 & 242 & 0.15 & 601 & 325 & 1.85 \\
\hline Kwan-Krolik $^{c}$. & 220 & 184 & 1.21 & 625 & 99 & 6.3 \\
\hline
\end{tabular}

a Observed equivalent width reduced to rest frame.

${ }^{b}$ Calculated for a covering factor of 0.2 and a cutoff for ionizing photons of 4 rydbergs from equations (1) or (2).

"In this case "observed" equivalent width means the equivalent width calculated by Kwan and Krolik 1979. The "predicted" equivalent width is that derived from equations (1) or (2) but with no cutoff as per Kwan and Krolik; $\alpha=1$. 
3 and the appropriate references. These are compared with those predicted for each quasar from the relation

$$
\begin{aligned}
\mathrm{EW}_{0}(L \alpha) & =\mathrm{CF} \frac{\lambda_{\mathrm{L} \alpha}}{\alpha}\left(\lambda_{\mathrm{L} \alpha} / \lambda_{\mathrm{LC}}\right)^{\alpha}\left(R^{\alpha}-1\right) \\
& =\mathrm{CF} \frac{1216}{\alpha}\left(\frac{4}{3}\right)^{\alpha}\left(R^{\alpha}-1\right) \AA \quad(\alpha \neq 0),
\end{aligned}
$$

where $\mathrm{EW}_{0}(\mathrm{~L} \alpha)$ is the equivalent width of $\mathrm{L} \alpha$ in the quasar rest frame, $\mathrm{CF}$ is the covering factor of the broad-line clouds, $\lambda_{\mathrm{L} \alpha}$ and $\lambda_{\mathrm{LC}}$ are the wavelengths of L $\alpha$ and the Lyman limit, $\alpha$ is the spectral index of the continuum source if the flux density $f_{\nu}$ is assumed to follow a power law $f_{\nu} \propto \nu^{\alpha}$, and $R$ is the ratio of the energy cutoff of ionizing photons to the energy of a photon at the Lyman limit. In evaluating equation (1), we have assumed that the slope $\alpha(\mathrm{L} \alpha, \mathrm{H} \alpha)$ determined between $\mathrm{L} \alpha$ and $\mathrm{H} \alpha$ is characteristic of the source beyond the Lyman limit.

The covering factor is the solid angle subtended by the broad-line clouds, as seen from the quasar continuum source, divided by $4 \pi$. This quantity can be estimated independently from the strength of the Lyman discontinuity in high-redshift quasars. Previous work (Oke 1974; Baldwin et al. 1976) suggest a covering factor $<0.1$ based on a lack of detection of Lyman continuum absorption in high-redshift quasars. Oke (1980) has reexamined this problem based on a larger sample of quasars and infers a covering factor of 0.150.25 . Based on these data, we therefore take 0.2 as an estimate of the covering factor for the present sample of quasars. This assumption is valid, of course, only if the same covering factor is applicable to both Oke's and the present samples of quasars.

The equivalent width of $L \alpha$ is sensitive to the highenergy cutoff selected; for $\alpha=-1$, the difference between a cutoff of 4 rydbergs and infinite energy is a $25 \%$ effect; for $\alpha=-0.5$, this difference is a $100 \%$ effect. A value of 4 rydbergs was chosen because this energy corresponds to the ionization energy of He II, and at this energy other elements begin to compete significantly with hydrogen for absorption of the continuum photons. Baldwin (1977a) has used the same limit in his estimates of the $\mathrm{L} \alpha$ equivalent widths.

The observed $\mathrm{L} \alpha$ equivalent widths reduced to the rest frames of the quasars are compared to those predicted from equation (1) in Table 6 . The average value of the ratio of observed to predicted $L \alpha$ equivalent widths is 0.24 for all nine quasars, and 0.18 if $1012+25$ (Ton 490) is excluded from the sample; $1012+25$ is anomalous in having very strong $\mathrm{H} \alpha$ and $\mathrm{L} \alpha$ lines and a very steep continuum. Since the observed equivalent widths are significantly less than predicted, we conclude that $L \alpha$ is weaker than expected by a factor 4-5.
The equivalent width of $\mathrm{H} \alpha$ can also be estimated from equation (1) and the continuum slope between $\mathrm{L} \alpha$ and $H \alpha$. If the $\mathrm{L} \alpha$ equivalent width is given by equation (1) and $\mathrm{L} \alpha / \mathrm{H} \alpha \approx 12$, the equivalent width of $\mathrm{H} \alpha$ is given by

$$
\begin{aligned}
\mathrm{EW}_{0}(\mathrm{H} \alpha) & =\frac{\mathrm{EW}_{0}(\mathrm{~L} \alpha)}{12}\left(\frac{\lambda_{\mathrm{H} \alpha}}{\lambda_{\mathrm{L} \alpha}}\right)^{2+\alpha} \\
& =2.43(5.4)^{\alpha} \mathrm{EW}_{0}(\mathrm{~L} \alpha),
\end{aligned}
$$

where $\mathrm{EW}_{0}(\mathrm{~L} \alpha)$ is the equivalent width of $\mathrm{L} \alpha$ derived in (1) and $\alpha$ is the spectral index of the continuum source between $\mathrm{H} \alpha$ and $\mathrm{L} \alpha$.

The observed equivalent widths of $\mathrm{H} \alpha$ reduced to the rest frames of the quasars are given in Table 6, and compared as the ratio of observed to predicted $\mathrm{H} \alpha$ equivalent widths. The average value of this ratio is found to be 2.4 for all quasars; if $1012+25$, where the predicted equivalent width of $\mathrm{H} \alpha$ is very small because of the steep continuum slope, is again deleted, this ratio is 1.6. The observed $\mathrm{H} \alpha$ equivalent width is thus stronger than that predicted by this simple approach, but is close enough to that predicted to conclude that $\mathrm{H} \alpha$ is not significantly enhanced with respect to that expected from the case $B$ values.

From this analysis it appears that, in the present sample of quasars, $\mathrm{L} \alpha$ is destroyed, rather than $\mathrm{H} \alpha$ enhanced. This conclusion disagrees with that reached by Baldwin (1977a), who drew just the opposite conclusion, i.e., $\mathrm{L} \alpha$ was not destroyed, but rather $\mathrm{H} \alpha$ was enhanced. The different conclusion comes from two effects; first, the covering factor taken by Baldwin was 0.1 , rather than 0.2; second, the observed widths of $\mathrm{L} \alpha$ found for the present sample of quasars are about half that taken to be typical by Baldwin. The combined effect of these two differences, neither of which is well established, changes the sense of the agreement from that found by Baldwin to the present conclusion. It should be noted that if the continuum slope steepens below the Lyman limit, the number of Lyman continuum photons and hence the $\mathrm{L} \alpha$ equivalent width would be overestimated. Thus the present conclusion must be regarded as tentative until observations of quasars below the Lyman limit are available.

\section{c) Comparison with Models}

\section{i) External Reddening}

Of the major classes of models invoked to explain the hydrogen line ratios in quasars, the implications of external reddening models are most readily apparent. The galactic latitudes tabulated in Table 5 are sufficiently high to rule out any significant contribution to the observed ratios from reddening in the Galaxy. If 


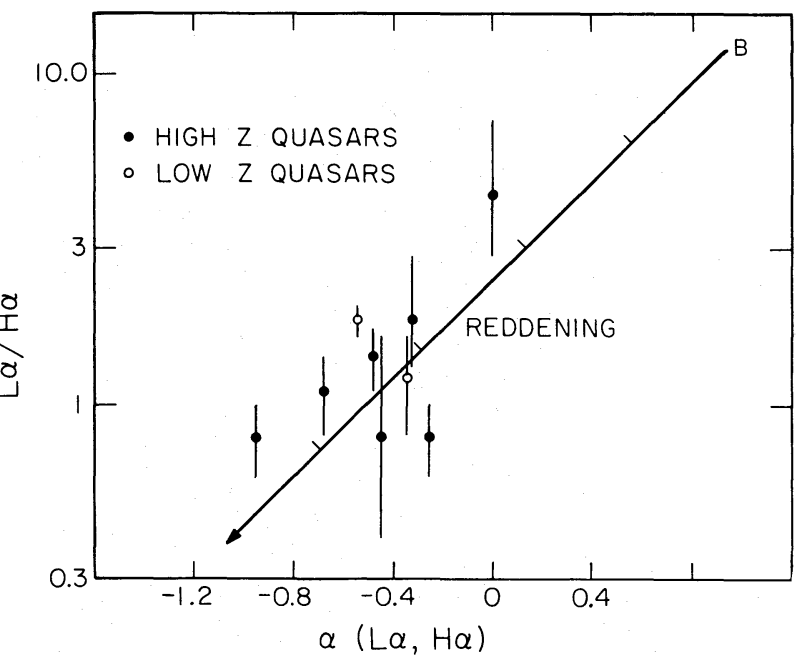

Fig. 6. - The $\mathrm{L} \alpha / \mathrm{H} \alpha$ line ratio plotted vs. continuum spectral index determined at those same wavelengths for all the quasars in Table 5. The arrow indicates the track that external reddening of an intrinsic quasar spectrum which affects the lines and continuum equally would take in this plot. The origin of this arrow is arbitrary, but has been drawn as the best fit to the observed data. The $B$ indicates the pure case $B$ recombination value of $\mathrm{L} \alpha / \mathrm{H} \alpha$. The successive tic marks indicate changes in $E(B-V)$ of 0.1 mag.

external, reddening must be "local" to the quasars because of the low values of $\mathrm{L} \alpha / \mathrm{H} \alpha$ shared by lowand high-redshift quasars.

Figures 6 and 7 show the relationship between the $\mathrm{L} \alpha / \mathrm{H} \alpha$ and $\mathrm{P} \alpha / \mathrm{H} \beta$ line ratios and the spectral index $\alpha$ of the continuum flux density $f_{v}$. For Figure 6 the spectral index is evaluated between the wavelengths of $\mathrm{L} \alpha$ and $\mathrm{H} \alpha$ using the continuum determined at those wavelengths. For Figure 7 the spectral index is evaluated between the wavelengths of $\mathrm{H} \beta$ and $\mathrm{P} \alpha$. If the reddening, though associated with the quasars, is external to the line-emitting region and is responsible for the anomalous ratios, the continuum must steepen as the $\mathrm{L} \alpha / \mathrm{H} \alpha$ ratio decreases. In Figure 6 , the arrow shows how a particular intrinsic quasar spectrum, reddened by various amounts, would change in the $\mathrm{L} \alpha / \mathrm{H} \alpha$ versus $\alpha$ plot if the emission lines and continuum were reddened by identical amounts of intervening material; the origin of this trajectory is arbitrary and has been adjusted to best fit the data. The apparent correlation between the observed data and the predicted slope shown in Figure 6 would suggest that external reddening of the continuum and lines together may be a controlling factor in determining the observed $\mathrm{L} \alpha / \mathrm{H} \alpha$ line ratios. If caused totally by external reddening, an average of $E(B-V) \sim 0.35 \mathrm{mag}$ would be needed to explain the observed deviation of the $\mathrm{L} \alpha / \mathrm{H} \alpha$ ratio from the case $B$ value. The correlation may, however, only be an artifact of the small number of objects in the present sample. In particular, if $1623+26(\mathrm{~L} \alpha / \mathrm{H} \alpha$ $=4, \alpha=0)$ is removed from Figure 6 , the correlation

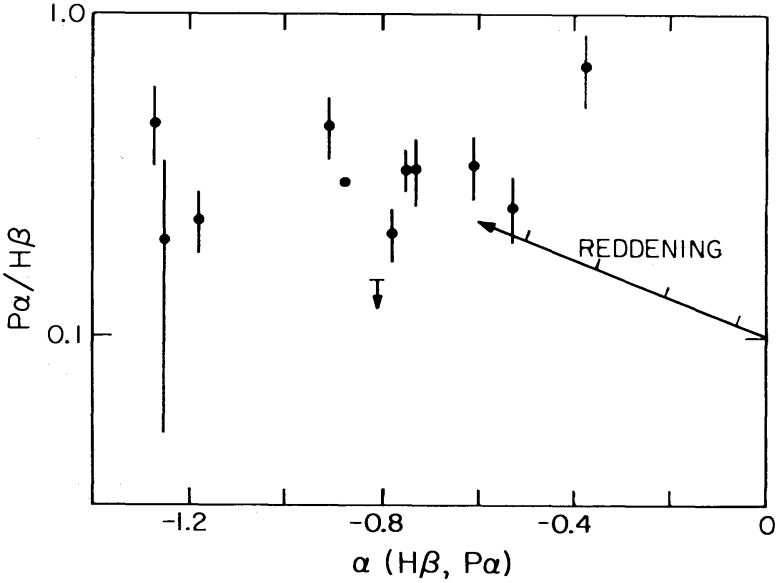

FIG. 7.- The $\mathrm{P} \alpha / \mathrm{H} \beta$ line ratio plotted vs. the continuum spectral index determined at those same wavelengths, for all the quasars in Table 4. The arrow indicates the track that external reddening of an intrinsic quasar spectrum which affects the lines and continuum equally would have in this plot. The origin of this arrow is arbitrary. The successive tic marks indicate changes in $E(B-V)$ of $0.1 \mathrm{mag}$.

between $\mathrm{L} \alpha / \mathrm{H} \alpha$ and spectral index disappears. Clearly this correlation must be substantiated by further observations of a larger sample of quasars.

In the context of this discussion, it should be noted that the line ratios of the Seyfert galaxies 3C 120 and Mrk 79 obey a reddening law indicated by the arrow in Figure 6 (Oke and Zimmerman 1979). Furthermore, when the total L $\alpha$ line is included for the radio galaxy 3C 390.3 (Ferland et al. 1979), the $\mathrm{L} \alpha / \mathrm{H} \alpha$ value deviates significantly from the trend, while if only the broad-line component of $\mathrm{L} \alpha$ is included, the agreement between the observed $\mathrm{L} \alpha / \mathrm{H} \alpha$ ratio and the arrow is satisfactory. Further evidence that reddenning is important comes from other observations. Netzer and Davidson (1979) have argued that the observed ratios of $\mathrm{He}$ II $\lambda \lambda 1640 / 4686$ and $\mathrm{O}_{\text {I }} \lambda \lambda 1304 / 8446$ are anomalously low in a significant sample of quasars and can be accounted for only by external reddening, since none of these lines should be significantly affected by radiative transfer effects in the broad-line regions.

While external reddening provides the simplest explanation of the observed correlation in Figure 6, two questions must be answered before such an explanation can be accepted. First, does other evidence support the reddening hypothesis, and second, can some other physical effect explain the correlation?

Other than the possible correlation presented in Figure 6, the direct evidence for reddening in the quasar spectra is weak, at best. Of the observed quasars, only $1012+25$ (Ton 490) shows weak evidence for absorption at $2175 \AA$ (Baldwin 1977a); this indicates that galactictype dust does not provide a major portion of the reddening. In the high-redshift quasar $1225+31$, Soifer et al. (1979) estimate an upper limit $E(\mathrm{~L} \alpha-\mathrm{H} \alpha) \approx 0.25$ 
mag corresponding to $E(B-V)<0.03 \mathrm{mag}$, based on the lack of a $2175 \AA$ band and a normal extinction curve; Boggess et al. (1979) place a similar limit on the extinction to 3C 273 from the ultraviolet spectrum of that quasar. Similar limits can be placed on reddening in the line of sight in the $2175 \AA$ band to the other high-redshift quasars in this study. In three cases, not observed in this program, Baldwin (1977a) found some evidence for redshifted absorption at $2175 \AA$, but the lack of detection of such a band in other quasars where the $\mathrm{L} \alpha / \mathrm{H} \alpha$ ratio is directly observed must cast doubt on such dust affecting the observed ratios.

In addition to this lack of direct evidence for reddening, the line ratios in the low-redshift quasars show little or no evidence for reddening. In Figure 5, the upper arrow shows the track of various amounts of reddening applied to the case $B$ recombination case. It is seen that the $\mathrm{P} \alpha / \mathrm{H} \alpha / \mathrm{H} \beta$ ratios follow a trajectory which is almost orthogonal to the way reddening should affect these ratios. Likewise, in Figure 7 , where the ratio of $\mathrm{P} \alpha / \mathrm{H} \beta$ is plotted versus continuum spectral index, the $\mathrm{P} \alpha / \mathrm{H} \beta$ ratio shows no evidence for a correlation suggestive of reddening affecting the lines and continuum. Indeed, many of the $\mathrm{P} \alpha / \mathrm{H} \beta$ ratios observed in quasars are less than the case $B$ value, and are therefore impossible to reconcile with an intrinsic ratio close to the case $B$ value coupled with reddening.

We must therefore conclude that if reddening external to the line-emitting region is the cause of the anomalous ultraviolet optical line ratios in quasars, the dust must be very unlike that known within the Galaxy. Properties that such dust must have are (i) no $2175 \AA$ absorption feature, (ii) no reddening between 0.5 and 2 $\mu \mathrm{m}$ (Figs. 5 and 7), and (iii) efficient extinction around L $\alpha$ (Fig. 6). Such dust can be devised by invoking, e.g., ad hoc dust grains with dimensions $\lesssim 0.01 \mu \mathrm{m}$, but composed of a material that does not possess a resonance at $\sim 2175 \AA$.

A further significant problem with this model is the lack of the reemission of the absorbed radiation at infrared wavelengths. In $1226+02$ (3C 273), the beststudied quasar, the bulk of the observed luminosity is emitted in or shortward of the ultraviolet portion of the spectrum (Neugebauer et al. 1979; Rieke and Low 1972; Hildebrand et al. 1977), but the $\mathrm{L} \alpha / \mathrm{H} \alpha$ ratio is lower than predicted by a factor of $\sim 10$. If this depression of the $\mathrm{L} \alpha / \mathrm{H} \alpha$ ratio is due to external reddening of both line and continuum radiation, the intrinsic luminosity of 3 C 273 should be $\sim 10$ times larger than the observed ultraviolet luminosity. Furthermore, if the material causing the extinction is distributed roughly spherically symmetrically with respect to $3 \mathrm{C} 273$, the extinction must be due to absorption rather than scattering, and this missing luminosity must be emitted in the infrared portion of the spectrum. None of the infrared observations reported to date shows any evidence for an infrared excess of such a magnitude.
The previous analysis, which indicates that the observed equivalent width of $\mathrm{L} \alpha$ is less by a factor of 2-5 than that predicted from the continuum slope determined between $\mathrm{H} \alpha$ and $\mathrm{L} \alpha$, also argues against significant reddening of both the lines and continuum. If the anomalous line ratios are caused by external dust, the observed equivalent width would, to first order, be the same as the intrinsic equivalent width. Likewise, the covering factor determined from the Lyman limit discontinuity would, to first order, be the same as the intrinsic covering factor. Because the observed continuum slope would be steeper than the intrinsic slope, the $\mathrm{L} \alpha$ equivalent width predicted from the observed continuum slope would be less than the true (observed) $\mathrm{L} \alpha$ equivalent width, in contradiction to the sense of the discrepancy found above.

As a quantitative example of this effect, if reddening decreases the $\mathrm{L} \alpha / \mathrm{H} \alpha$ ratio by a factor of 10 , the continuum slope between $\mathrm{H} \alpha$ and $\mathrm{L} \alpha$ is decreased by $\Delta \alpha \sim 1.4$, and the equivalent width of $L \alpha$ predicted from equation (1) is a factor of $\sim 3.5$ smaller than the intrinsic (observed) equivalent width. Thus the discrepancy between the ratio of observed to predicted $\mathrm{L} \alpha$ equivalent widths found from the data and that expected if external reddening were the cause of the observed $\mathrm{L} \alpha / \mathrm{H} \alpha$ line ratios, is roughly a factor of 15 .

This leaves the question of whether there is an alternative explanation for the apparent correlation between the $\mathrm{L} \alpha / \mathrm{H} \alpha$ ratio and the continuum spectral index. Puetter (private communication) has suggested a possible explanation of this effect based on the heating of the line-emitting regions by the ionizing radiation. The $\mathrm{L} \alpha$ and $\mathrm{H} \alpha$ lines are both sufficiently optically thick that the line ratio is determined by the Planck limit at the kinetic temperature of the gas (c.f. Zirin 1978), which should be determined to some extent by the input ionizing spectrum. The variation should be in the sense shown in Figure 6, namely, that the flatter ionizing spectra (increasing spectral index) produce a hotter gas, and hence a larger $\mathrm{L} \alpha / \mathrm{H} \alpha$ ratio. Clearly this model must be studied in further detail.

The observations are thus contradictory with respect to the importance of reddening by intervening dust. The data in Figure 6 are consistent with a significant amount of reddening of both the line and continuum sources. In contrast, the data of Figures 5 and 7 are not consistent with such a model, nor is the absence of the $2175 \AA$ absorption feature or the apparent weakness of L $\alpha$ compared to the continuum. On the basis of the above discussion we favor the conclusion that extinction by intervening dust is not primarily responsible for the observed anomalous line ratios.

\section{ii) Internal Reddening}

Another reddening model postulates that dust internal to the broad-line-emitting region absorbs reso- 
nantly scattered in $\mathrm{L} \alpha$ radiation and can explain several features of the $\mathrm{L} \alpha / \mathrm{H} \alpha$ line ratios. First, the optical depths in $\mathrm{L} \alpha$ provide sufficient path length for dust to absorb these photons, while not altering the other lines significantly. Second, the lack of observed $2175 \AA$ absorption is explained since the dust does not obscure the continuum source. Third, there is no need to increase the quasar intrinsic luminosity by a large factor and yet hide it from observation. The luminosity in L $\alpha$ that is absorbed by dust in this model is less than $10 \%$ of the quasar luminosity.

While this model has advantages, it also has drawbacks. First, as London (1979) pointed out, the environment in the quasar emission line regions is exceedingly harsh, and whether dust grains could survive is questionable. Second, detailed calculations by Ferland and Netzer (1979) and Shuder and MacAlpine (1979) have not succeeded in producing observed line ratios (notably $\mathrm{He}$ II $\lambda \lambda 1640 / 4686$, and $\mathrm{L} \alpha / \mathrm{H} \alpha$ ) with the inclusion of dust in the line-emitting regions. Indeed, both works conclude that a mechanism in addition to internal dust absorption is needed to account for the observed line ratios in quasars.

The line ratio data presented here are contradictory with respect to this model. If the model is correct, there should be no correlation between the line ratios and continuum slope; the data of Figure 7 are thus consistent with this model. Second, the internal dust model clearly predicts that $\mathrm{L} \alpha$ should be destroyed, since it is absorbed by dust in the line-emitting regions. This sense is consistent with the analysis of the present data which suggests that $\mathrm{L} \alpha$ is indeed destroyed in the line-emitting regions. The data of Figure 5 do not, however, support this model. In particular, as previously noted, any decrease of the $\mathrm{P} \alpha / \mathrm{H} \alpha$ and $\mathrm{P} \alpha / \mathrm{H} \beta$ ratios below the case $B$ value is hard to reconcile with the presence of dust, either internal or external to the line-emitting region. Finally, the possible correlation noted in Figure 6, if substantiated, would also speak against such a model.

\section{iii) Optical Depth and Collisional Models}

Since the models involving internal or external reddening are hard-pressed to explain the observed hydrogen line ratios, it is necessary to consider alternative models. Models have been proposed which invoke radiative transfer and collisional excitation effects in the lines to explain the observed line ratios. These models, by their very complex nature, are difficult to compare to the observations presented here. Additionally, the models published to date have concentrated on predicting line ratios, and have not in general attempted to predict the equivalent widths of the observed lines. Such calculations would be accessible to observational tests through data such as those presented in Table 6, and can provide further insight into the size scales of the line-emitting regions.
The most detailed models published to date, those of Kwan and Krolik (1979), produce line ratios in general agreement with those observed. Kwan and Krolik report basically only one model, and this has a Balmer decrement larger than typically observed. They also predict the photon equivalent width of $\mathrm{L} \alpha$, i.e., the ratio of $\mathrm{L} \alpha$ photons emitted to Lyman continuum photons absorbed. The $\mathrm{L} \alpha$ and $\mathrm{H} \alpha$ equivalent widths predicted by Kwan and Krolik are compared with the case $B$ values from equations (1) and (2) in Table 6. Their results indicate that $\mathrm{H} \alpha$ is enhanced over the case B prediction rather than $\mathrm{L} \alpha$ being destroyed. This seems to disagree with the observational result that suggests $\mathrm{L} \alpha$ destruction. It is interesting to note that $1012+25$ (Ton 490) agrees quite well with the Kwan and Krolik model, and the discrepancy could in fact be accounted for by a $30 \%$ change in covering factor. Ton 490 also shows the steepest continuum of the quasars in Table 6, and is in fact observationally similar to the model calculated by Kwan and Krolik.

The most significant trend found in the present data that should be reproduced in any variable model is the variation of the $\mathrm{P} \alpha / \mathrm{H} \alpha$ ratio with the $\mathrm{H} \alpha / \mathrm{H} \beta$ ratio. The data of Figure 5 show a reasonably tight correlation in the sense that $\mathrm{H} \alpha$ appears to increase with respect to both $\mathrm{P} \alpha$ and $\mathrm{H} \beta$, while the $\mathrm{P} \alpha / \mathrm{H} \beta$ ratio seems to be roughly constant. The simplest interpretation of this variation is that the Balmer lines are optically thin, while $\mathrm{H} \alpha$ is enhanced by collisional excitation (Krolik and McKee 1978). Unfortunately, the models that reproduce the $\mathrm{L} \alpha / \mathrm{H} \alpha$ ratios via a combination of large optical depths and collisional excitation (Kwan and Krolik 1979; Canfield and Puetter 1980b) imply large optical depths in the Balmer lines, and so are incompatible with the simple interpretation of Figure 5. Previous calculations of the effects of large Balmer optical depths on the $\mathrm{P} \alpha / \mathrm{H} \alpha / \mathrm{H} \beta$ ratios in regions of high densities such as in the broad-line regions of quasars (Netzer 1975; Krolik and McKee 1978) show a variation that is suggestive of a nearly constant $\mathrm{P} \alpha / \mathrm{H} \alpha$ line ratio, while the $\mathrm{H} \alpha / \mathrm{H} \beta$ ratio varies over a range of 3 to $\sim 8$. This behavior is not found in the present data except for the case of $2141+$ 174, but the calculations of Netzer (1975) show that for very large optical depths in $\mathrm{H} \alpha$, the line ratios return to close to case B values, so that perhaps collisions can produce the same net effects as in the low optical depth limit. Much more extensive models, such as those described by Canfield and Puetter (1980b) that treat in detail the effects of the transfer of line radiation in a very dense, optically thick region, are clearly needed to understand whether such processes can truly reproduce the observed variations.

\section{SUMMARY AND CONCLUSIONS}

From an extensive optical infrared study of hydrogen lines in low- and high-redshift quasars we have 
found:

1. The $\mathrm{P} \alpha / \mathrm{H} \alpha$ ratios in low-redshift quasars are distributed around the case $B$ value with a tendency toward values lower than that predicted by case $B$. The $\mathrm{H} \alpha / \mathrm{H} \beta$ values in these same quasars are generally greater than the case $B$ value. The trend in the $\mathrm{P} \alpha / \mathrm{H} \alpha / \mathrm{H} \beta$ ratios is for the $\mathrm{P} \alpha / \mathrm{H} \alpha$ ratio to decrease as $\mathrm{H} \alpha / \mathrm{H} \beta$ increases.

2. The low values of the $\mathrm{L} \alpha / \mathrm{H} \alpha$ ratio compared to the case $B$ value are confirmed for a large sample of high-redshift quasars. A possible correlation between the $\mathrm{L} \alpha / \mathrm{H} \alpha$ ratio and continuum spectral index of the continuum is found in the sense that steeper continuum spectra are associated with smaller $\mathrm{L} \alpha / \mathrm{H} \alpha$ line ratios.

3. $\mathrm{L} \alpha$ is apparently destroyed rather than $\mathrm{H} \alpha$ being enhanced. This conclusion is based on an analysis of the observed equivalent widths of $\mathrm{L} \alpha$ and $\mathrm{H} \alpha$ combined with the flux expected from available ionizing photons and an independent determination (Oke 1980) of the broad-line covering factor in quasars.

4. We conclude that reddening external to the emission-line regions cannot satisfactorily explam all the observed hydrogen line ratios. The current observations do not support the model of internal dust destroying resonantly scattered $\mathrm{L} \alpha$, but such a model cannot be ruled out on the basis of the present data.

5. Models of the emission-line regions that apply radiative transfer and collisonal excitation effects in the lines appear to be necessary to explain the current observations. The decrease of the $\mathrm{P} \alpha / \mathrm{H} \alpha$ ratio with increasing $\mathrm{H} \alpha / \mathrm{H} \beta$ ratio is the most significant correlation found form the present data that any valid model of the line-emitting regions must explain.

We thank our observing assistants Gary Tuton, Juan Carrasco, Bruce Cuney, and Howard Larning, and S. Beckwith, I. Gatley, A. Hyland, J. Mould, D. Nadeau, and $K$. Sellgren for assistance in obtaining these observations, R. Green for providing positions of quasars in advance of publication, R. Puetter and H. E. Smith for discussing their unpublished data with us, J. Krolik and R. Puetter for discussing the theoretical implications of the observations, and J. Krolik and K. Davidson for a careful reading of the manuscript. This work was supported by NASA and NSF grants.

\section{REFERENCES}

Baldwin, J. A. 1975, Ap. J., 201, 26. 1977a, M.N.R.A.S., 178, 67P $1977 b, A p$. J., 214, 679.

Baldwin, J. A., and Netzer, H. 1978, Ap. J., 226, 1.

Baldwin, J. A., Rees, M. J., Longair, M. S., and Perryman, M. A. C. 1978, Ap. J. (Letters), 226, L57.

Baldwin, J. A., Smith, H. E., Burbidge, E. M., Hazard, C., Murdoch, H. S., and Jauncey, D. L. 1976, Ap. J. (Letters), 206, L83.

Boggess, A., et al. 1979, Ap. J. (Letters), 230, L131.

Brocklehurst, M. 1972, M.N.R.A.S., 157, 211.

Canfield, R. C., and Puetter, R. C. 1980a, Ap. J. (Letters) 236, L7.

\section{$1980 b$, preprint}

Davidsen, A. F., and Hartig, G. F. 1978, Proc. COSPAR/IAU Symposium in X-Ray Astronomy, ed. W. A. Braity and L. E. Peterson, (New York: Pergamon).

Davidsen, A. F., Hartig, G. F., and Fastie, W. G. 1977, Nature, 269, 203.

Davidson, K. 1972, Ap. J., 171, 213.

Davidson, K., and Netzer, H. 1979, Rev. Mod. Phys., 51, 715.

Ferland, G. J., and Netzer, H. 1979, Ap. J., 229, 274.

Ferland, G. J., Rees, M. J., Longair, M. A., and Perryman, M. A. C. 1979, M.N.R.A.S., 187, 65P.

Grandi, S. A., and Phillips, M. M. 1979, Ap. J., 232, 659.

Grasdalen, G. L. 1976, Ap. J. (Letters), 208, L11.

Hildebrand, R. H., Whitcomb, S. E., Winston, R., Steining, R. F., Harper, D. A., and Moseley, S. H. 1977, Ap. J., 216, 698.

Hyland, A. R., Becklin, E. E., and Neugebauer, G. 1978, Ap. J. (Letters), 220, L73.
Krolik, J. H., and McKee, C. F. 1978, Ap. J. Suppl., 37, 459.

Kwan, J., and Krolik, J. H. 1979, Ap. J. (Letters), 233, L91.

London, R. 1979, Ap. J., 228, 8.

Netzer, H. 1975, M.N.R.A.S., 171, 395.

Netzer, H., and Davidson, K. 1979, M.N.R.A.S., 187, 871.

Netzer, H., Wills, B. J., Uomoto, A. K., Rybski, P. M., and Tull, R. G. 1979, Ap. J. (Letters), 232, L155.

Neugebauer, G. Oke, J. B., Becklin, E. E., and Matthews, K. 1979, Ap. J., 230, 79.

Oke, J. B. 1974, Ap. J., 189, L47 1980 , in preparation.

Oke, J. B., and Zimmerman, B. 1979, Ap. J. (Letters), 230, L13.

Osmer, P. A., and Smith, M. G. 1977, Ap. J., 213, 607.

Pengelly, R. M., and Seaton, M. J. 1964, M.N.R.A.S. 127, 165

Puetter, R. C., Smith, H. E., Soifer, B. T., Willner, S. P., and Pipher, J. L. 1978, Ap. J. (Letters), 226, L53.

Puetter, R. C., Smith, H. E., and Willner, S. P. 1979, Ap. J. (Letters), 227, L5.

Puetter, R. C., Smith, H. E., Willner, S. P., and Pipher, J. L. 1981, Ap. J., 2A3, 345.

Rieke G. L., and Low, F. J. 1972, Ap. J. (Letters), 176, L95.

Sandage, A. 1972, Ap. J., 178, 1.

Scargle, J. D., Caroff, L. V., and Tarter, C. B. 1974, Ap. J., 189, 181.

Shuder, J. M., and MacAlpine, G. M. 1979, Ap. J., 230, 348.

Soifer, B. T., Oke, J. B., Matthews, K., and Neugebauer, G. 1979, Ap. J. ( Letters) 227, L1.

Sramek, R. A., and Weedman, D. W. 1978, Ap. J., 221, 468.

Zirin, H. 1978, Ap. J. (Letters), 222, L105.

K. Matthews, B. T. Solfer: and G. Neugebauer, Division of Physics, Mathematics, and Astronomy, Downs Laboratory 320-47, California Institute of Technology, Pasadena, CA 91125

J. B. OKe; Division of Physics, Mathematics, and Astronomy, Robinson Laboratory 105-24, California Institute of Technology, Pasadena, CA 91125 